\title{
Epoxidation of 1,5,9-cyclododecatriene with hydrogen peroxide under phase-transfer catalysis conditions: influence of selected parameters on the course of epoxidation
}

\section{Grzegorz Lewandowski ${ }^{1} \oplus \cdot$ Marcin Kujbida $^{2} \odot \cdot$ Agnieszka Wróblewska $^{2}([)$}

Received: 14 December 2020 / Accepted: 3 March 2021 / Published online: 12 March 2021

(c) The Author(s) 2021

\begin{abstract}
This work presents the studies on the epoxidation of 1,5,9-cyclododecatriene (CDT) with hydrogen peroxide as the oxidizing agent, under conditions of the phase transfer catalysis (PTC), and with the following catalytic system: $\mathrm{H}_{2} \mathrm{WO}_{4} / \mathrm{H}_{3} \mathrm{PO}_{4} /\left[\mathrm{CH}_{3}(\right.$ $\left.\left.\mathrm{CH}_{2}\right)_{7}\right]_{3} \mathrm{CH}_{3} \mathrm{~N}^{+} \mathrm{HSO}_{4}{ }^{-}$(compounds were mixed at the ratio of 2:1:1). The influence of the following parameters on the course of this process was investigated: catalyst content, molar ratio of $\mathrm{H}_{2} \mathrm{O}_{2}: \mathrm{CDT}$, temperature and type of solvent. The highest yield of 1,2-epoxy-5,9-cyclododecadiene (ECDD) $(54.9 \mathrm{~mol} \%)$, at the conversion of CDT reached $72.3 \mathrm{~mol} \%$, was obtained at the temperature of $50{ }^{\circ} \mathrm{C}$, for the catalyst content of $0.45 \mathrm{~mol} \%$ (in relation to the introduced CDT), for the molar ratio of $\mathrm{H}_{2} \mathrm{O}_{2}$ :CDT 1.5:1, with toluene as the solvent and after the reaction time of $30 \mathrm{~min}$. Considering the he obtained results and numerous applications of ECDD, further research should be developed to provide a more efficient and environmentally friendly way of obtaining this compound.
\end{abstract}

Grzegorz Lewandowski

grzegorz.lewandowski@zut.edu.pl

$\bowtie$ Agnieszka Wróblewska agnieszka.wroblewska@zut.edu.pl

1 Faculty of Chemical Technology and Engineering, Department of Chemical Organic Technology and Polymeric Materials, West Pomeranian University of Technology Szczecin, Pułaskiego 10, 70-322 Szczecin, Poland

2 Faculty of Chemical Technology and Engineering, Department of Catalytic and Sorbent MateriaIs Engineering, West Pomeranian University of Technology Szczecin, Pułaskiego 10, 70-322 Szczecin, Poland 


\section{Graphic abstract}

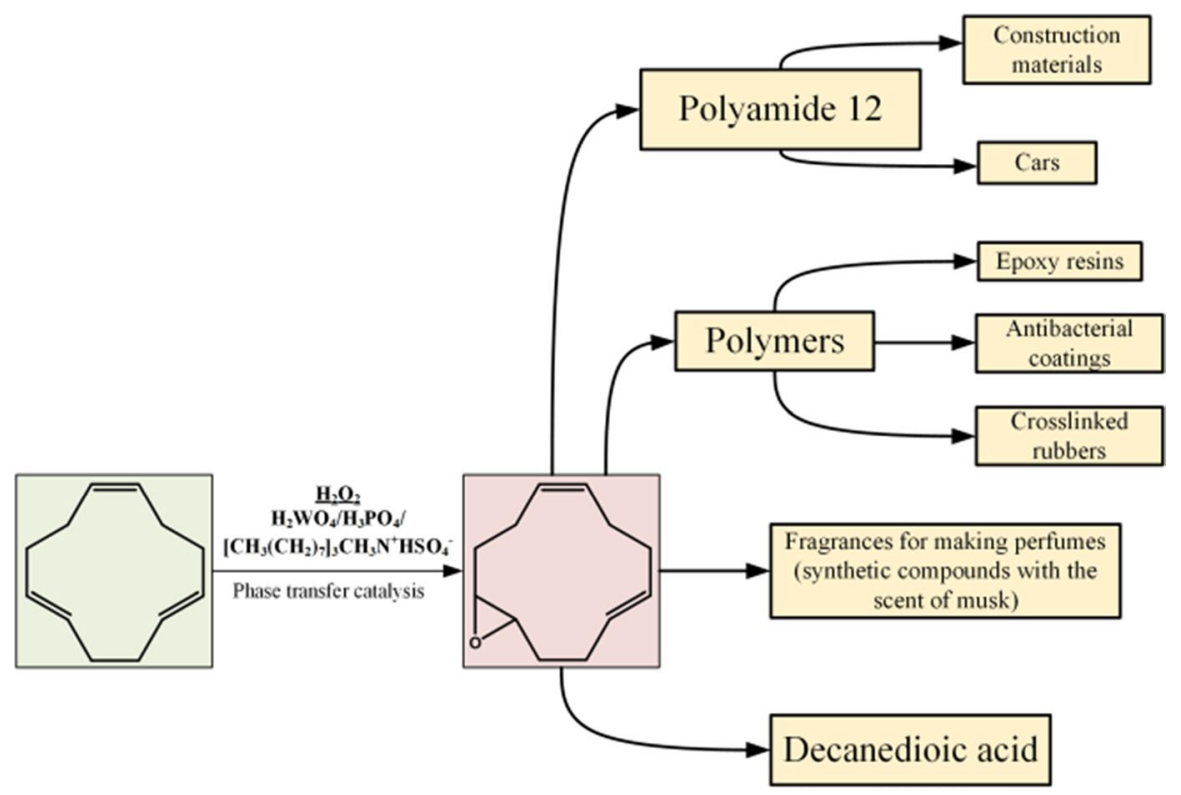

Keywords 1,5,9-Cyclododecatriene · Epoxidation · 1,2-Epoxy-5,9-

cyclododecadiene $\cdot$ Hydrogen peroxide $\cdot$ Phase-transfer catalysis (PTC)

\section{Introduction}

1,2-Epoxy-5,9-cyclododecadiene (ECDD), due to the presence of two double bonds in the molecule and an oxirane ring, is used as an additive increasing the degree of crosslinking of polymers. Polymers with its addition can be used as elements of lithium-ion batteries [1-4], as well as capacitors [5]. It can also be used in the production of anti-static coatings [6,7], cross-linked acrylic rubber resistant to oils at high temperature [8] or antibacterial coatings [9]. Pure ECDD [10], as well as its derivative, i.e. 4,8-cyclododecadien-1-one [11] can be used as ingredients for perfumes with musky and earthy scents. At present, studies are also conducted on the use of this compound as an intermediate in the synthesis of cyclododecanol (CDOL) and cyclododecanone (CDON), from which 1,10-decanedicarboxylic acid (DDDA) and laurolactam can be obtained. Both of them are widely used as the raw materials in the production of polyesters and polyamides (Fig. 1) [12].

Laurolactam is a raw material in the production of polyamide 12 (PA12), which has the smallest share of amide groups in its molecule, which determines its characteristic properties. Its high hydrophobicity causes significant dimensional stability in conditions of changing humidity. PA12 is also known for its exceptional impact strength even at low temperatures. It is also resistant to most 


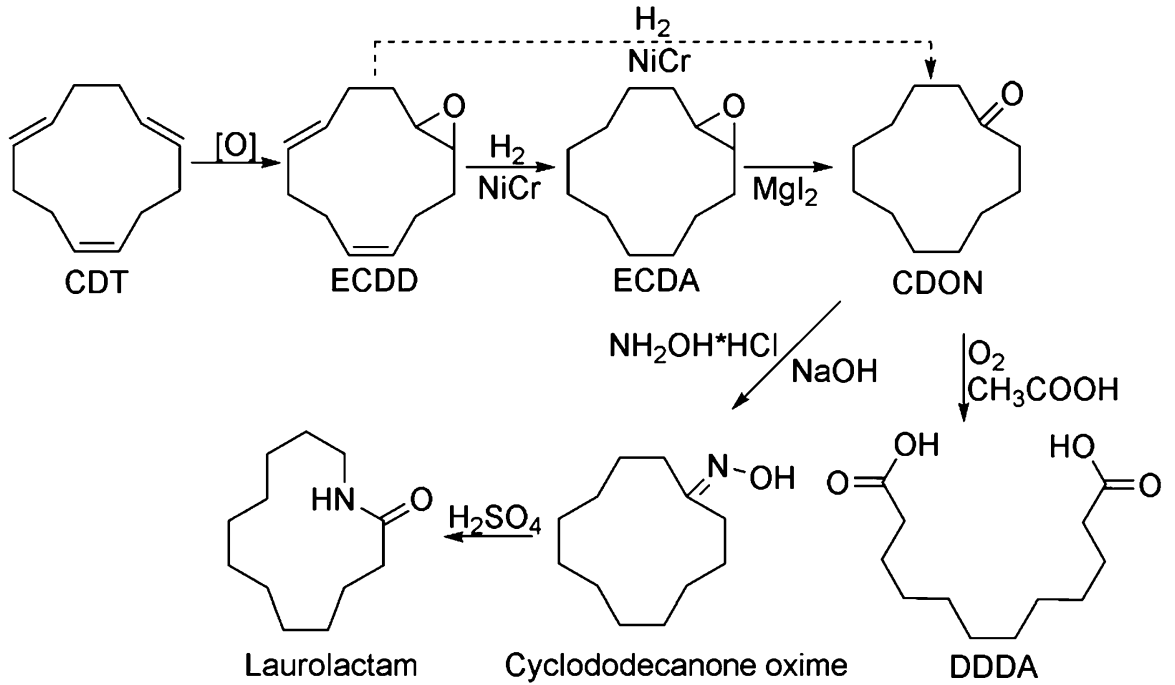

Fig. 1 Method of obtaining of laurolactam and decanedicarboxylic acid from CDT (CDT 1,5,9-cyclododecatriene, ECDD 1,2-epoxy-5,9-cyclododecadiene, ECDA epoxycyclododecane, $C D O N$ cyclododecanone, $D D D A$ 1,10-decanedicarboxylic acid)

chemicals: greases, fats, oils, fuels, solvents, salt solutions and others [13]. The above-mentioned properties make it an important construction material in the production of automotive fuel lines. Its key role in the automotive industry is demonstrated by the huge size of problems with the availability of raw materials for the production of car parts, caused by an accident at the Evonik factory in the city of Marl in Germany producing CDT, a raw material in the production of PA12 [14-16]. Fig. 2 shows the methods used to obtain ECDD.

Like most epoxides, ECDD can also be obtained in reacting of the appropriate olefin with an organic peracid, this reaction is sometimes named as the reaction of Prilezhaev [17]. During epoxidation of CDT using commercially available peracetic acid (PES-40), which is a mixture of: $40 \%$ peracetic acid, $40 \%$ acetic acid, 5\% hydrogen peroxide, $14 \%$ water and $1 \%$ sulfuric acid (VI) (the molar ratio CDT:PES-40 was 1:1.1), the desired product (ECDD) was obtained with the yield of $84 \%$ (in relation to the introduced CDT) and within $4 \mathrm{~h} \mathrm{[18].} \mathrm{The} \mathrm{reac-}$ tion was carried out by dropping PES-40 into a solution of CDT in chloroform (the ratio $1: 3 \mathrm{~m} / \mathrm{m}$ ), which contained anhydrous sodium carbonate (the molar ratio CDT: sodium carbonate $1: 1$ ) and at the temperature of $5{ }^{\circ} \mathrm{C}$. A modification of this method is the application of hydrogen peroxide as an oxidant and as an organic catalyst of an aliphatic acid, with a dissociation constant ranging from $5.0 \times 10^{-6}$ to $1.0 \times 10^{-4}$ [19]. The advantage of this method is the reduction of the reaction time in comparison to the method presented above (from 4 to $1 \mathrm{~h}$ ). By this method very high selectivity (in relation to reacted hydrogen peroxide) to monoepoxide (up to $98.8 \%$ at the conversion of $\mathrm{H}_{2} \mathrm{O}_{2} 83.7 \%$ and at the conversion CDT 21\%) is obtained. However, the increasing in the molar excess of CDT in relation to hydrogen peroxide $(4: 1$ instead of $1: 1.1)$ means that the maximum 


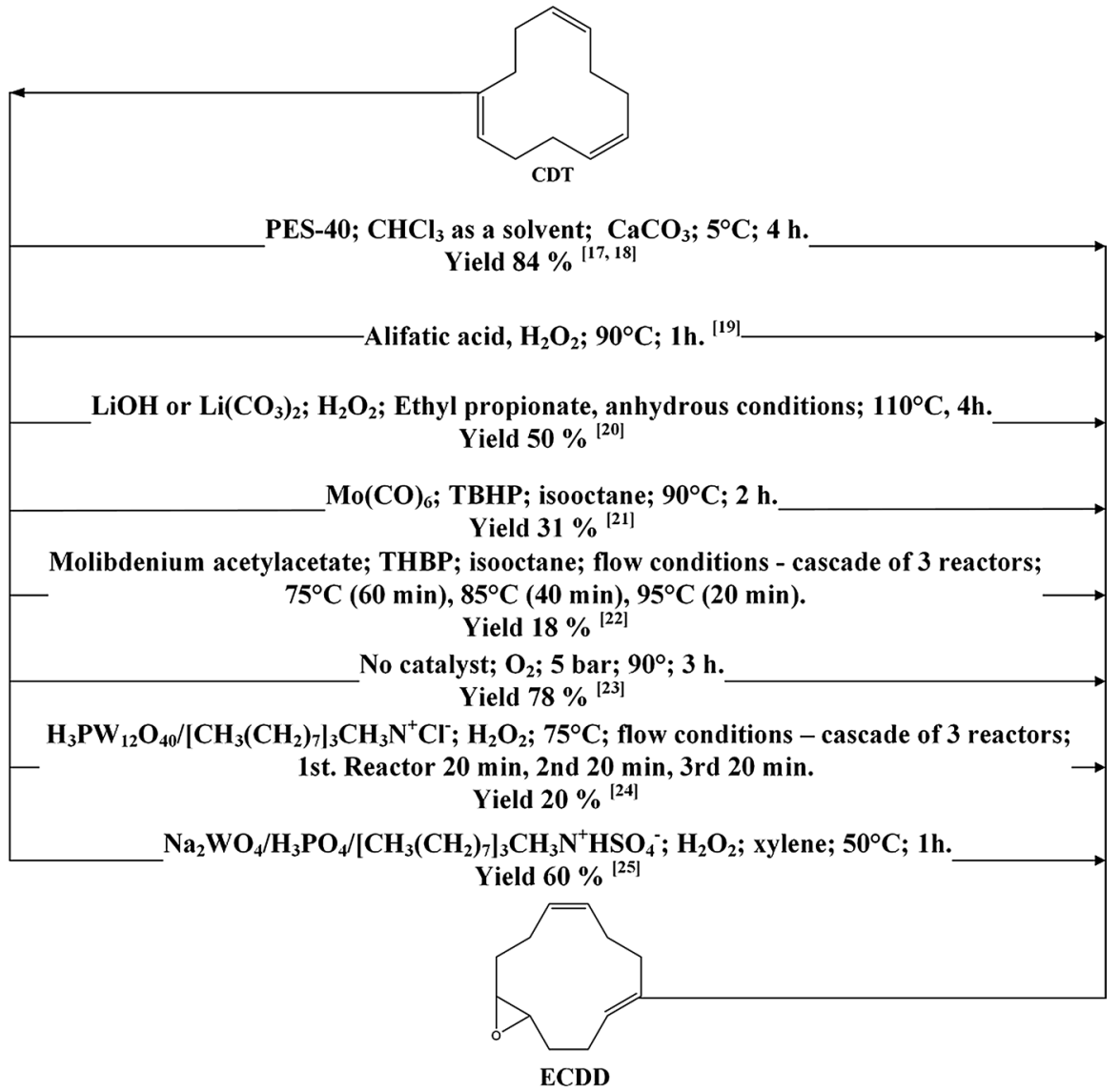

Fig. 2 Methods used for obtaining of ECDD

of CDT conversion can be only $25 \mathrm{~mol} \%$, which significantly reduces the yield of ECDD in relation to the introduced olefin.

Epoxidation of CDT with hydrogen peroxide using lithium as the catalyst is also possible. The highest ECDD yields (calculated on the introduced CDT) were obtained using lithium carbonate or hydroxide $-50 \%$ and $51 \%$, respectively. The reaction was carried out for $4 \mathrm{~h}$ at $110^{\circ} \mathrm{C}$. Anhydrous ethyl propionate was used as the solvent. In order to provide anhydrous reaction conditions, the introduced and formed water was discharged from the reaction medium in the form of a water: ethyl propionate azeotrope. Long reaction times and high reaction temperatures, as well as the need to provide an anhydrous environment, make this reaction inconvenient for industrial applications [20].

Lewandowski et al. [21] described the method of the obtaining of ECDD, consisting of epoxidation of CDT with tert-butyl hydroperoxide (TBHP) with the concentration of $20-80 \%$ and in the presence of a homogeneous molybdenum catalyst (molybdenum hexacarbonyl, molybdenyl acetylacetonate). The epoxidation of CDT 
is carried out in the temperature range of $70-90{ }^{\circ} \mathrm{C}$, at the atmospheric pressure, with the molar ratio of CDT to TBHP from $1: 1$ to $1: 5$, at the concentration of the appropriate solvent amounted to 13-70\% (iso-octane, decane, nonane, di-tert-butyl peroxide, 1,2-dichloroethane) and for the reaction time from 1 to $5 \mathrm{~h}$. The best results were obtained for the epoxidation performed at $90{ }^{\circ} \mathrm{C}$ for the reaction time $2 \mathrm{~h}$ and using isooctane as the solvent in the amount of about $22 \mathrm{wt} \%$ in relation to the mass of the reaction mixture. The catalyst was molybdenum hexacarbonyl and it was used in the molar ratio to TBHP of $5 \times 10^{-4}$. The molar ratio of CDT to TBHP was 2.94:1. In this way, the selectivity of transformation of CDT to ECDD was $99.6 \mathrm{~mol} \%$, at the conversion of CDT and TBHP amounted to $31.1 \mathrm{~mol} \%$ and $99.8 \mathrm{~mol} \%$, respectively.

From the patent specification prepared by Lewandowski et al. [22] is also known the continuous way of conducting the epoxidation of CDT, using the same reagents, in a cascade of flow reactors with intensive mixing and dosing of CDT in the mixture with the catalyst to the first stage of the cascade and a gradual introduction of the TBHP solution in the solvent to the first and subsequent stages of the cascade, with which in the next stages of the cascade a gradient temperature rise is maintained. The best results were obtained when epoxidation was carried out in a three-stage reactor cascade. In subsequent stages of the cascade, higher and higher temperatures were maintained, while reducing the residence time of the reaction mixture in them. Temperatures and residence times in successive stages of the cascade were as follows: $75^{\circ} \mathrm{C}$ and $60 \mathrm{~min}, 85^{\circ} \mathrm{C}$ and $40 \mathrm{~min}, 95{ }^{\circ} \mathrm{C}$ and $20 \mathrm{~min}$. TBHP was introduced into each cascade stage as a $6 \mathrm{M}$ solution in isooctane also containing dissolved catalyst $-0.007 \mathrm{M}$ molybdenum acetoacetate. In this way, the selectivity of transformation of CDT to ECDD was $91 \mathrm{~mol} \%$, while the conversion of CDT and TBHP amounted to $19.8 \mathrm{~mol} \%$ and $98 \mathrm{~mol} \%$, respectively.

Mahajan et al. [23] investigated the kinetics of non-catalysed oxidation of CDT with air oxygen. The reaction proceeded according to a radical mechanism, and its main by-product at lower temperatures and for shorter reaction times was cyclododecatryl hydroperoxide. When the temperature of epoxidation was increased, this hydroperoxide decomposed, increasing the selectivity of transformation to ECDD. The authors managed to obtain satisfactory CDT conversions and selectivity of transformation of CDT to ECDD, respectively: 84.5 and $92 \%$, by conducting the reaction in a stainless-steel reactor for $3 \mathrm{~h}$. The disadvantage of this method is the use of pure oxygen as an oxidizer, which creates a danger of an explosion.

Nobuyuki et al. patented the method of continuous obtaining of CDT under PTC conditions. The catalyst consisted of tungsten compounds (e.g. tungstic acid; sodium, potassium, lithium or ammonium tungstate; sodium, potassium, ammonium dodecatungstate; phosphotungstic acid, sodium phosphotungstate; silicotungstic acid; sodium silicotungstate) and ammonium or pyridinium salt, with possible addition of a mineral acid. Their method of carrying out this process consisted of conducting reactions in three subsequent reactors, with raw materials being fed only to the first of them. This process can be carried out at moderate temperatures $\left(20-120^{\circ} \mathrm{C}\right)$ and at the atmospheric, reduced or increased pressure. The best results were obtained when the process was carried out sequentially in three reactors for 20 min and at $75{ }^{\circ} \mathrm{C}$. CDT, phosphotungstic acid, methyltrioctylammonium chloride 
and $60 \%$ aqueous hydrogen peroxide were introduced into the first reactor (the molar ratio of CDT: $\mathrm{H}_{2} \mathrm{O}_{2}$ was about 4:1). After leaving the first reactor, the conversion of CDT and hydrogen peroxide was $15.3 \%$ and $68.8 \%$, respectively. The oil phase after the reaction contained $76.4 \mathrm{wt} \%$ of CDT and $21.9 \mathrm{wt} \%$ of ECDD. This corresponded to the conversion of CDT reached $21.9 \mathrm{~mol} \%$ and the selectivity of ECDD amounted to $93.2 \mathrm{~mol} \%$. The hydrogen peroxide conversion was $98.8 \%$. After vacuum distilling the oil phase at $3.0 \mathrm{kPa}$ and at $118^{\circ} \mathrm{C}$, CDT with the purity of $98.4 \%$ was recovered (it contained $1.4 \mathrm{wt} \%$ of ECDD). After distillation at $130{ }^{\circ} \mathrm{C}$ and $1.3 \mathrm{kPa}$ pressure of the oil phase remaining after CDT recovery, ECDD was obtained with the purity of $99.6 \%$ [24].

Lewandowski et al. also described the method of the obtaining of ECDD [25], which involved epoxidation of CDT with hydrogen peroxide, in the presence of the organic solvent, in the presence of sodium tungstate and ortho-phosphoric acid as the epoxidation catalyst, and methyltrioctylammonium bisulfate as the phase transfer catalyst. The process was carried out at $30-70{ }^{\circ} \mathrm{C}$, for $1-2 \mathrm{~h}$ and using intensive mixing. The molar ratio of sodium tungstate to ortho-phosphoric acid was 0.5-4.0:1. The molar ratio of hydrogen peroxide to sodium tungstate was 37-300:1, and the molar ratio of hydrogen peroxide to CDT was $0.75-1.5: 1$. As the organic solvent $m-, o-$, or $p$-xylene was used or a mixture of these solvents. The molar ratio of methyltrioctylammonium hydrogen sulfate to sodium tungstate was $0.3-0.5: 1$. In the process obtained relatively high ECDD yields in relation to the introduced CDT (18.3-59.7\%), in comparison to classical CDT epoxidation methods, carried out with tert-butyl hydroperoxide and peracetic or perbenzoic acid. The disadvantage of this method of epoxidation of CDT is the relatively low activity of the sodium tungstate/ortho-phosphoric acid catalytic system, which requires a relatively high temperature, reaching even $70{ }^{\circ} \mathrm{C}$ and a long reaction time, even $2 \mathrm{~h}$, to achieve full conversion of hydrogen peroxide.

The described methods for obtaining ECDD have disadvantages that limit their use in industrial conditions. Further research is needed to develop an environmentally friendly technology of obtaining this compound. Oxidation of CDT with hydrogen peroxide under the conditions of phase transfer catalysis (PTC) may be such a technology, however, the variants of this method described above have a number of drawbacks such as: long reaction time and low CDT conversion.

The purpose of the research presented in this work is to investigate the effect of changing the selected parameters (catalyst content, molar ratio CDT: $\mathrm{H}_{2} \mathrm{O}_{2}$, temperature and type of solvent) on the yield of ECDD and the conversion of CDT in the process of the epoxidation of CDT with hydrogen peroxide. The process will be carried out under PTC conditions, using the catalytic system consisting of tungsten (VI) acid, ortho-phosphoric acid (V) and methyltrioctylammonium hydrogen sulfate. No studies are available in the literature on the influence of the above-mentioned parameters on the course of the CDT epoxidation process using this complex catalytic system. We anticipate that there may be visible an increase in the reaction rate of ECDD formation, due to the higher acidity of the catalyst (replacement of the sodium salt of tungsten (VI) acid with tungsten (VI) acid), compared to the process carried out according to the patent [25]. Due to the numerous and new applications of ECDD (Nylon 12 and the component of musk fragrance compositions described 
in this work), it is desirable to develop a new, efficient and environmentally friendly method of obtaining this compound.

\section{Experimental section}

\section{Raw materials}

Reactions and determinations were carried out using the following reagents (without further purification): $c, t, t-1,5,9$-cyclododecatriene (98\%, Aldrich); hydrogen peroxide (30\% aqueous solution, Chempur); methyltrioctylammonium hydrogen sulfate ( $\geq 95 \%$, Aldrich); ortho-phosphoric acid (85\%, POCh Gliwice); dodecane ( $\geq 99 \%$, Aldrich); toluene (analytical grade, Chempur); sodium thiosulfate (analytical weight for preparation of $0.1 \mathrm{n}$ solution, Chempur); potassium iodide (analytical grade, POCh), chloroform (analytical grade, Chempur); ethyl acetate (analytical grade, Chempur). The ECDD standard for GC was obtained and purified in our laboratory.

\section{Method of CDT epoxidation}

Into a glass reactor with a capacity of $25 \mathrm{~cm}^{3}$ equipped with a thermometer, a mechanical stirrer and a reflux condenser, tungsten (VI) acid, methyltrioctylammonium hydrogen sulfate, ortho-phosphoric acid (V) in the form of a $10 \mathrm{wt} \%$ aqueous solution and hydrogen peroxide were added successively. The reactor contents were mixed at $1000 \mathrm{rpm}$ for $15 \mathrm{~min}$ at a room temperature. After this time, a mixture consisting of CDT, solvent and dodecane as an internal standard was added to the reactor, its composition at the beginning was additionally determined by a gas chromatography method (GC). The reactor was then placed in a water bath having the appropriate temperature and its contents was mixed at a speed of $1000 \mathrm{rpm}$. Samples of the organic phase were taken after the reaction time: 5, 10, 15 and $30 \mathrm{~min}$, and their composition was analyzed by the GC method. After the reaction time $60 \mathrm{~min}$, the contents of the reactor were poured into a plastic tube, weighed and centrifuged at $4{ }^{\circ} \mathrm{C}$ for $15 \mathrm{~min}$ at $5000 \mathrm{rpm}$. The aqueous and organic phases were separated and weighed. In both phases, the content of unreacted hydrogen peroxide was determined using the iodometric method, and the composition of the organic phase was additionally determined by the GC method.

\section{Parameters of chromatographic analyses}

Quantitative analyses of the tested samples was carried out using the internal standard method (dodecane) using a Thermo-Finigan Trace GC 2000 chromatograph with a Chrom-Card for Trace GC data processing program. A DB-5 capillary column, $\mathrm{J} \& \mathrm{~W}$ Scientific, $30 \mathrm{~m} \times 0.25 \mathrm{~mm} \times 0.25 \mu \mathrm{m}$ was used for the analyses. The sample chamber temperature was $200{ }^{\circ} \mathrm{C}$ and the detector $250{ }^{\circ} \mathrm{C}$. The oven's temperature program was as follows: isothermally $50{ }^{\circ} \mathrm{C}$ for $2.5 \mathrm{~min}$, increase to $250{ }^{\circ} \mathrm{C}$ at the rate of $20^{\circ} \mathrm{C} / \mathrm{min}$ and isothermally $250{ }^{\circ} \mathrm{C}$ for $2.5 \mathrm{~min}$. Carrier gas pressure (helium) 
was changed as follows: initially $75 \mathrm{kPa}$, then an increase at a rate of $5 \mathrm{kPa} / \mathrm{min}$ to $150 \mathrm{kPa}$. Gas flows in the detector: air $350 \mathrm{~cm}^{3} / \mathrm{min}$, hydrogen $35 \mathrm{~cm}^{3} / \mathrm{min}$ and helium (compensating gas) $10 \mathrm{~cm}^{3} / \mathrm{min}$. Internal calibration curve was made for ECDD using the following ECDD/dodecane weight ratios: $0.12 ; 0.25 ; 0.58 ; 1.00$; 2.00 to get a directional coefficient of 0.9789 and a coefficient of $\mathrm{R}^{2}$ equal to 0.9947 . The curve for CDT was made for the weight ratio CDT/dodecane: $0.12 ; 0.25 ; 0.50$; 1.00; 2.00 and its directional coefficient and $\mathrm{R}^{2}$ were equal to 0.8431 and 0.9973 , respectively. The content (in wt $\%$ ) of the components of the reaction mixture was calculated from the following formula:

$$
c_{i}=\alpha_{i} * \frac{A_{i}}{A_{w}} * \frac{m_{w}}{m_{p}} * 100 \%
$$

Here: $\alpha_{i}$-directional factor of the calibration curve of the' i' component, $A_{i}, A_{w}$ - peak areas of the established substance and standard, $m_{w}, m_{p}$-mass: standard and sample $(\mathrm{g})$. The organic phase samples taken from the reactor were analyzed without additional dilution.

\section{The method of calculating the results}

The yield of ECDD $\left(\mathrm{W}_{\mathrm{ECDD}}\right)$, and the conversions of CDT $\left(\mathrm{K}_{\mathrm{CDT}}\right)$ and $\mathrm{H}_{2} \mathrm{O}_{2}$ $\left(\mathrm{K}_{\mathrm{H}_{2} \mathrm{O}_{2}}\right)$, were calculated using the following equations:

$$
\begin{gathered}
W_{E C D D}=\frac{n_{E C D D}}{n_{0, C D T}} * 100 \% \\
K_{C D T}=\frac{n_{0, C D T}-n_{C D T}}{n_{0, C D T}} * 100 \% \\
K_{\mathrm{H}_{2} \mathrm{O}_{2}}=\frac{n_{0, \mathrm{H}_{2} \mathrm{O}_{2}}-n_{\mathrm{H}_{2} \mathrm{O}_{2}}}{n_{0, \mathrm{H}_{2} \mathrm{O}_{2}}} * 100 \%
\end{gathered}
$$

$n_{E C D D}, n_{C D T}$-number of CDT moles: initial and at the time of measurement (mmol), $n_{E C D D}$-number of ECDD moles at the time of measurement (mmol), $n_{0, \mathrm{H}_{2} \mathrm{O}_{2}}, n_{\mathrm{H}_{2} \mathrm{O}_{2}}$ - the number of moles $\mathrm{H}_{2} \mathrm{O}_{2}$ : at the beginning of the reaction and at the time of measurement (mmol).

\section{Results and discussion}

\section{Influence of the catalyst content}

The first stage of the study was to examine the effect of the content of the catalyst in the form of the catalyst system: tungsten (VI) acid/ortho-phosphoric (V) acid/ methyltrioctylammonium hydrogen sulfate on the course of the epoxidation of CDT. 
Usually, the increase in the catalyst content increases the rate of reaction, but in case of the epoxidation of CDT, the desired product (ECDD) can be formed first in a series of follow-up reactions. There is also the possibility of simultaneous epoxidation of two or three double bonds and direct formation of diECDD and triECDD (Fig. 3) [26]. The formation of triECDD as a result of the simultaneous oxidation of two double bonds ECDD or direct oxidation of diECDD can not be excluded. There is no clear information on the mechanism of this process in the literature.

Thus, an increase in catalyst content may cause a reduction in the selectivity of the transformation CDT to ECDD due to the further epoxidation of ECDD and diECDD or the parallel epoxidation of two or three double CDT bonds. In this regard, it may be that there is an optimal catalyst content at which diECDD and triECDD are formed in a minimal amount.

In order to study the effect of this parameter on the kinetics of CDT epoxidation with hydrogen peroxide, a series of studies were performed with increasing content of the tungsten (VI) acid/ortho-phosphoric (V) acid/methyltrioctylammonium hydrogen sulfate $(0.17 ; 0.30 ; 0.45 ; 0,55 ; 1.19 \mathrm{~mol} \%$ CDT calculated per atom W) and with other parameters constant: the weight ratio CDT to toluene (solvent) 1:1, the molar ratio of $\mathrm{H}_{2} \mathrm{O}_{2}$ :CDT $1.5: 1$, while the molar ratios of the catalyst components were as follows: $\mathrm{H}_{2} \mathrm{WO}_{4}: \mathrm{H}_{3} \mathrm{PO}_{4}:\left[\mathrm{CH}_{3}\left(\mathrm{CH}_{2}\right)_{7}\right]_{3} \mathrm{CH}_{3} \mathrm{~N}^{+} \mathrm{HSO}_{4}{ }^{-}$2:1:1.

Table 1 presents the values of the conversion of CDT (the values of the yields of ECDD are also given in brackets) for reactions carried out with different catalyst contents.

Changes in conversion of CDT as a function of reaction time for increasing catalyst contents are shown in Fig. 4 and Table 1.

The results presented in Fig. 4 and Table 1 clearly show that the conversion of CDT is directly proportional to the catalyst content-the higher the content of the catalyst, the higher the conversion of CDT (after the given reaction time). It can be seen that the conversion of CDT in three syntheses with the highest catalyst content $(1.19 ; 0.55$; and $0.45 \mathrm{~mol} \%$ CDT) tends to be similar after $60 \mathrm{~min}$ of reaction performing (about $93 \mathrm{~mol} \%$ ). In case of reactions carried out with

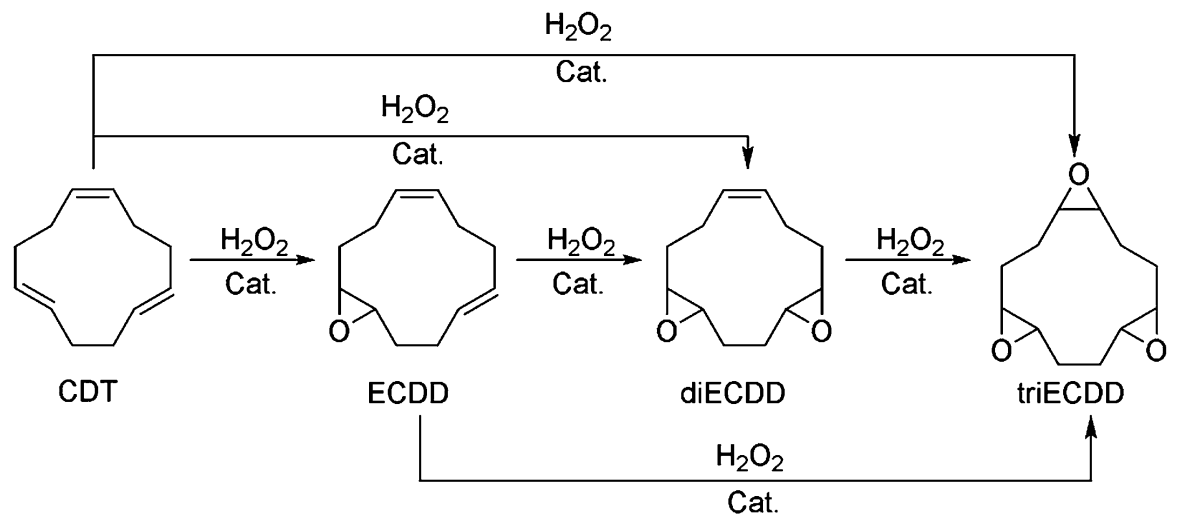

Fig. 3 Possible ways of ECDD, diECDD and triECDD obtaining 
Table 1 The values of the conversion of CDT (the values of the yields of ECDD are also given in brackets) for reactions carried out with different catalyst contents ${ }^{\mathrm{a}}$

\begin{tabular}{|c|c|c|c|c|c|}
\hline \multirow[t]{2}{*}{ Time $(\min )$} & \multicolumn{5}{|c|}{ Catalyst content (mol\% CDT) } \\
\hline & 0.17 & 0.30 & 0.45 & 0.55 & 1.19 \\
\hline 0 & $0(0)$ & $0(0)$ & $0(0)$ & $0(0)$ & $0(0)$ \\
\hline 5 & $14.5(11.4)$ & $22.6(14.8)$ & $25.5(18.2)$ & $35.6(26.1)$ & $41.0(24.1)$ \\
\hline 10 & $17.4(15.4)$ & $30.7(23.0)$ & $36.6(30.6)$ & $48.6(37.2)$ & $61.2(36.8)$ \\
\hline 15 & $24.9(22.6)$ & $40.5(31.6)$ & $48.1(39.5)$ & $59.5(47.0)$ & $76.8(46.4)$ \\
\hline 30 & $40.7(35.1)$ & $58.7(43.6)$ & $72.3(54.9)$ & $83.3(53.2)$ & $91.7(41.0)$ \\
\hline 60 & $66.0(54.5)$ & $86.2(45.5)$ & $91.7(46.2)$ & $93.3(45.0)$ & $92.9(41.0)$ \\
\hline $60^{\mathrm{b}}$ & 48.7 & 77.8 & 94.9 & 98.9 & 99.2 \\
\hline
\end{tabular}

${ }^{\text {a }}$ The reaction conditions were the same as those given in the title in Fig. 4

${ }^{\mathrm{b}}$ The values of conversion of $\mathrm{H}_{2} \mathrm{O}_{2}$
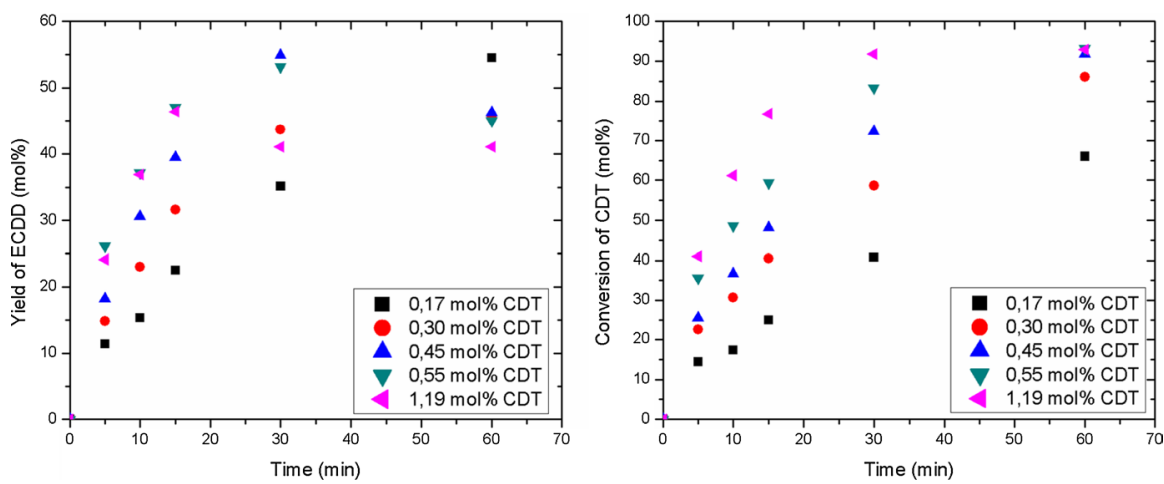

Fig. 4 Drawings showing the conversion of CDT and the yield of ECDD as a function of time for reactions with different catalyst contents. Reaction conditions: temperature $50{ }^{\circ} \mathrm{C}$, stirring speed $1000 \mathrm{rpm}$, solvent content (toluene) $50 \mathrm{wt} \%$, the molar ratios $\mathrm{H}_{2} \mathrm{WO}_{4}: \mathrm{H}_{3} \mathrm{PO}_{4}:\left[\mathrm{CH}_{3}\left(\mathrm{CH}_{2}\right)_{7}\right]_{3} \mathrm{CH}_{3} \mathrm{~N}^{+} \mathrm{HSO}_{4}^{-}$2:1:1

a smaller catalyst content $(0.17$ and $0.30 \mathrm{~mol} \% \mathrm{CDT})$, the conversions of CDT after the reaction time of $60 \mathrm{~min}$ are lower and amount to 66.0 and $86.2 \mathrm{~mol} \%$, respectively. However, analyzing the course of changes in the conversion of CDT as a function of reaction time, it can be assumed that also in these cases, after appropriate extension of the reaction time, a similar conversion of CDT would be obtained as in other syntheses. Limitation of CDT conversion to approx. $93 \mathrm{~mol} \%$ is caused by the depletion of $\mathrm{H}_{2} \mathrm{O}_{2}$, which is transformed not only in the reaction of ECDD formation, but also diECDD. $\mathrm{A}_{2} \mathrm{O}_{2}$ decomposition reaction can not be excluded either.

Changes in the yield of ECDD yield as a function of reaction time shown in Fig. 4 also depend on the content of the catalyst, but they are not as easy to interpret as changes in the conversion of CDT. This is due to the aforementioned nature of CDT epoxidation-this is a reaction in which the by-products such as 
diECDD and triECDD are formed as a result of subsequent epoxidation of ECDD and diECDD, respectively, or of parallel CDT epoxidation.

It should be emphasized that with the technological parameters used in this work, virtually no formation of triECDD was found. The only by-product was diECDD, whose amount in the post-reaction mixture increases as the conversion of CDT increases.

It is visible during the analysis the changes of yield of ECDD as a function of reaction time that when the conversion of CDT exceeds about $70 \mathrm{~mol} \%$ (reactions with content of the catalyst $0.45,0.55$ and 1.19 mol CDT) yields of ECDD are decreasing. This may indicate a greater likelihood of diECDD formation due to a post-epoxidation of ECDD than parallel CDT epoxidation. Probably during the epoxidation of CDT, for the appropriate reaction time, there is already enough amount of ECDD in the reaction mixture that it becomes more readily available than CDT and may undergo further oxidation. The highest yield of ECDD was obtained by carrying out the epoxidation for $30 \mathrm{~min}$ with the catalyst content of $0.45 \mathrm{~mol} \%$ CDT.

The conversion of hydrogen peroxide (Table 1) is also dependent on the catalyst content. For the smallest catalyst content $(0.17 \mathrm{~mol} \% \mathrm{CDT})$, the conversion of $\mathrm{H}_{2} \mathrm{O}_{2}$ after $60 \mathrm{~min}$ of reaction carrying out is only $48.7 \mathrm{~mol} \%$. Increasing in the catalyst content to $0.30 \mathrm{~mol} \%$ CDT increases the conversion of $\mathrm{H}_{2} \mathrm{O}_{2}$ to $77.8 \mathrm{~mol} \%$. Further increasing in the catalyst content (to $0.45 \mathrm{~mol} \% \mathrm{CDT}$ ) increases the conversion of $\mathrm{H}_{2} \mathrm{O}_{2}$ to $94.9 \mathrm{~mol} \%$.

For reactions carried out with the catalyst content of over $0.55 \mathrm{~mol} \% \mathrm{CDT}$, the conversion of hydrogen peroxide is almost complete.

\section{Influence of the molar ratio of $\mathrm{H}_{2} \mathrm{O}_{2}$ :CDT}

The next stage of our research was checking the influence of the molar ratio of $\mathrm{H}_{2} \mathrm{O}_{2}$ :CDT on the results of the epoxidation. This parameter is important because the molar ratio of $\mathrm{H}_{2} \mathrm{O}_{2}$ :CDT directly determines the side reactions of oxidation of the second and third double bonds in CDT molecule and the formation of larger amounts of diECDD and triECDD.

Five molar ratios of $\mathrm{H}_{2} \mathrm{O}_{2}$ :CDT were checked: $0.75 ; 1 ; 1.25 ; 1.5$ and $2: 1$. Other reaction parameters were as follows: temperature $50{ }^{\circ} \mathrm{C}$, catalyst content $0.55 \mathrm{~mol} \%$ CDT, and toluene as the solvent.

On the graph of changes in the conversion of CDT as a function of time (Fig. 5) it can be seen that for each reaction time studied, an increase in the $\mathrm{H}_{2} \mathrm{O}_{2}$ :CDT molar ratio causes an increase in the conversion of CDT. In the range of reaction times from 5 to $30 \mathrm{~min}$ and for the molar ratios of $\mathrm{H}_{2} \mathrm{O}_{2}$ :CDT 1-1.5:1, very similar values of conversions of CDT were obtained at individual reaction times. Extending the reaction time to $60 \mathrm{~min}$, however, reveals significant differences in conversion of CDT as a function of the molar ratio of $\mathrm{H}_{2} \mathrm{O}_{2}$ :CDT. The lowest conversion of CDT $(64.1 \mathrm{~mol} \%)$ was obtained for the molar ratio of $\mathrm{H}_{2} \mathrm{O}_{2}$ :CDT 0.75 , the highest $(98.1 \mathrm{~mol} \%)$ at the molar ratio of $\mathrm{H}_{2} \mathrm{O}_{2}:$ CDT $2: 1$. At intermediate molar ratios of $\mathrm{H}_{2} \mathrm{O}_{2}$ :CDT (in the range of $1-1.5: 1$ ) the conversions 

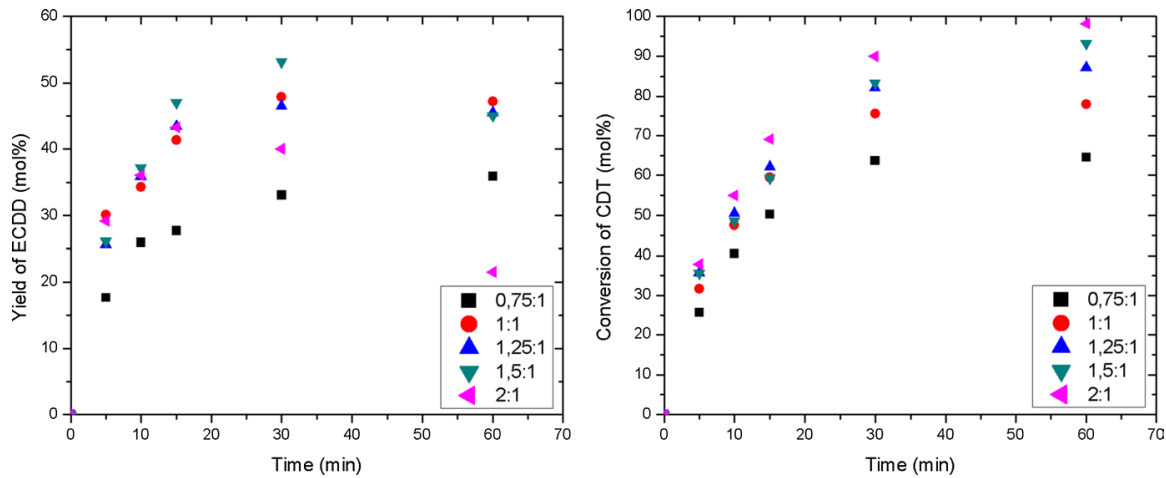

Fig. 5 Drawings showing the conversion of CDT and the yield of ECDD as a function of time for reactions with different molar ratios of $\mathrm{H}_{2} \mathrm{O}_{2}$ :CDT. Reaction conditions were the same as those given in the title in Fig. 4

of CDT in the range of 78-93.3 mol\% were obtained. Considering the conversion of $\mathrm{H}_{2} \mathrm{O}_{2}$ after 60 min of reaction carrying out (Table 2), which was close to the total for most reactions of this series of tests, it should be emphasized that as the molar ratio of $\mathrm{H}_{2} \mathrm{O}_{2}$ :CDT increases from $0.75: 1$ to $2: 1$ the conversion of CDT also increases, however, the efficiency of $\mathrm{H}_{2} \mathrm{O}_{2}$ utilization is gradually decreasing. This is especially visible when comparing the syntheses carried out at the highest (2:1) and stoichiometric (1:1) molar ratio of $\mathrm{H}_{2} \mathrm{O}_{2}$ :CDT. The conversions of $\mathrm{H}_{2} \mathrm{O}_{2}$ obtained for both syntheses are similar, but the yield of ECDD for the molar ratio of $\mathrm{H}_{2} \mathrm{O}_{2}$ :CDT 2:1 is definitely lower. In this synthesis, the conversion of $\mathrm{H}_{2} \mathrm{O}_{2}$ was as much as $92.1 \mathrm{~mol} \%$, which indicates that more than $50 \%$ of $\mathrm{H}_{2} \mathrm{O}_{2}$ has undergone side reactions-formation of diECDD and unproductive degradation (decomposition of $\mathrm{H}_{2} \mathrm{O}_{2}$ into water and molecular oxygen).

Table 2 The values of the conversion of CDT (the values of the yields of ECDD are also given in brackets) for reactions carried out with a different molar ratios of $\mathrm{H}_{2} \mathrm{O}_{2}: \mathrm{CDT}^{\mathrm{a}}$

\begin{tabular}{llllll}
\hline Time (min) & \multicolumn{5}{l}{ Molar ratio of $\mathrm{H}_{2} \mathrm{O}_{2}: \mathrm{CDT}$} \\
\cline { 2 - 6 } & $0.75: 1$ & $1: 1$ & $1.25: 1$ & $1.5: 1$ & $2: 1$ \\
\hline 0 & $0(0)$ & $0(0)$ & $0(0)$ & $0(0)$ & $0(0)$ \\
5 & $25.7(17.7)$ & $31.6(30.1)$ & $35.6(25.6)$ & $35.6(26.1)$ & $37.8(29.2)$ \\
10 & $40.4(25.9)$ & $47.6(34.3)$ & $50.5(35.9)$ & $48.6(37.2)$ & $55.0(36.0)$ \\
15 & $50.3(27.7)$ & $59.6(41.3)$ & $62.1(43.4)$ & $59.5(47.0)$ & $69.0(43.3)$ \\
30 & $63.7(33.1)$ & $75.6(47.9)$ & $82.2(46.5)$ & $83.3(53.2)$ & $89.9(40.0)$ \\
60 & $64.5(35.9)$ & $78.0(47.2)$ & $87.2(45.5)$ & $93.3(45.0)$ & $98.1(21.5)$ \\
$60^{\text {b }}$ & 99.4 & 99.7 & 99.0 & 98.9 & 92.1 \\
\hline
\end{tabular}

${ }^{\mathrm{a}}$ The reaction conditions were the same as those given in the title in Fig. 4

${ }^{b}$ The values of conversion of $\mathrm{H}_{2} \mathrm{O}_{2}$ 
In case of the yield of ECDD depicted as a function of reaction time in Fig. 5, it can be seen that up to $15 \mathrm{~min}$ for all tested molar ratios of $\mathrm{H}_{2} \mathrm{O}_{2}$ :CDT, except 0.75:1, similar values of the yield of ECDD were obtained for all studied reaction time. In 30 min there is visible a division into reactions with still increasing (the molar ratio of $\mathrm{H}_{2} \mathrm{O}_{2}$ :CDT $0.75: 1$ ), stabilized (the molar ratios of $\mathrm{H}_{2} \mathrm{O}_{2}$ :CDT $1: 1$ and $1.25: 1$ ) and decreasing (the molar ratios of $\mathrm{H}_{2} \mathrm{O}_{2}$ :CDT 1.5:1 and 2:1) values of the yield of ECDD. For the molar ratio of $\mathrm{H}_{2} \mathrm{O}_{2}$ :CDT 2:1, a significant reduction in the yield of ECDD occurs after $15 \mathrm{~min}$ of reaction performing (from 43.3 to $21.5 \mathrm{~mol} \%$ ), and for the molar ratio of $\mathrm{H}_{2} \mathrm{O}_{2}$ :CDT 1.5:1 after $30 \mathrm{~min}$ (from 53.2 to $45 \mathrm{~mol} \%$ ). This is most likely caused by the subsequent reaction of oxidation of ECDD to diECDD. The decrease in the yield of ECDD is also noticeable for reactions in which the oxidant was used in a smaller excess or equimolar to olefin (1.25 and 1:1). However, in these cases the decrease in the yield of ECDD is very small, by 1.0 and $0.7 \mathrm{~mol} \%$, respectively. From a technological point of view, it does not seem beneficial to use a large excess of oxidant relative to the stoichiometric amount of CDT. At high molar ratios of $\mathrm{H}_{2} \mathrm{O}_{2}$ :CDT relatively high yields of ECDD can be obtained. The highest yield of ECDD amounted to $53.2 \mathrm{~mol} \%$ for the molar ratio of $\mathrm{H}_{2} \mathrm{O}_{2}$ :CDT 1.5:1 and after 30 min of reaction carrying out, however, both the $\mathrm{H}_{2} \mathrm{O}_{2}$ utilization rate and the selectivity of transformation of CDT to ECDD are not optimal.

\section{Influence of temperature}

The kinetics of the CDT epoxidation with hydrogen peroxide was studied by carrying out the reaction at temperatures $40,50,60$ and $70{ }^{\circ} \mathrm{C}$ and with constant values other parameters (catalyst content $0.55 \mathrm{~mol} \% \mathrm{CDT}$, the molar ratio of $\mathrm{H}_{2} \mathrm{O}_{2}$ :CDT 1.5:1; toluene as the solvent). The results of these studies are presented in Fig. 6 and Table 3.

On the graph showing the conversion of CDT as a function of reaction time (Fig. 6) it can be seen that it is highly dependent on the reaction temperature. It seems interesting to compare the values of conversion of CDT after 5 min of running
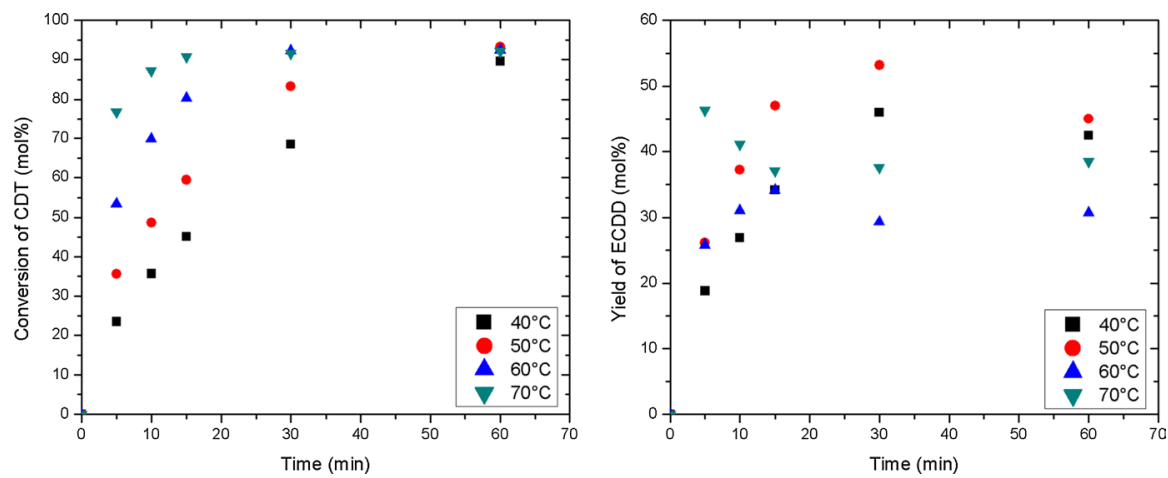

Fig. 6 Drawings showing the conversion of CDT and the yield of ECDD as a function of time for reactions carried out at different temperatures. Reaction conditions were the same as those given in the title in Fig. 4 
Table 3 The values of the conversion of CDT (the values of the yields of ECDD are also given in brackets) for reactions carried out with a different temperatures $^{\mathrm{a}}$

\begin{tabular}{lllll}
\hline Time (min) & \multicolumn{4}{l}{ Temperature } \\
\cline { 2 - 5 } & $40^{\circ} \mathrm{C}$ & $50^{\circ} \mathrm{C}$ & $60^{\circ} \mathrm{C}$ & $70^{\circ} \mathrm{C}$ \\
\hline 0 & $0(0)$ & $0(0)$ & $0(0)$ & $0(0)$ \\
5 & $23.5(18.8)$ & $35.6(26.1)$ & $53.4(25.8)$ & $76.8(46.3)$ \\
10 & $35.8(26.8)$ & $48.6(37.2)$ & $69.9(31.0)$ & $87.2(41.1)$ \\
15 & $45.2(34.1)$ & $59.5(47.0)$ & $80.3(34.1)$ & $90.7(37.1)$ \\
30 & $68.5(46.0)$ & $83.3(53.2)$ & $92.2(29.3)$ & $91.7(37.6)$ \\
60 & $89.6(42.4)$ & $93.3(45.0)$ & $92.4(30.6)$ & $92.2(38.5)$ \\
$60 \mathrm{~b}$ & 86.7 & 98.9 & 99.1 & 99.6 \\
\hline
\end{tabular}

${ }^{\text {a }}$ The reaction conditions were the same as those given in the title in Fig. 4

${ }^{b}$ The values of conversion of $\mathrm{H}_{2} \mathrm{O}_{2}$

the process at different temperatures. At the temperature of $40{ }^{\circ} \mathrm{C}$, the conversion of CDT is only $23.5 \mathrm{~mol} \%$, while at the temperatures 50,60 and $70{ }^{\circ} \mathrm{C}$ it increases successively to $35.6,53.4$ and $76.8 \mathrm{~mol} \%$. Such a large increase in the conversion of CDT indicates the dominant effect of temperature on the CDT epoxidation process. However, such large differences between the values of the conversion of CDT at individual temperatures occur at the initial stage of the reaction (5-15 min) and disappear in $60 \mathrm{~min}$ of the reaction carrying out. It should be emphasized that for all temperatures a very similar final maximum value of conversion of CDT of about 90-93 mol\% is obtained, however, its achievement requires definitely different reaction times. For syntheses carried out at 40 and $50{ }^{\circ} \mathrm{C}$, maximum of the conversion of CDT is obtained only after $60 \mathrm{~min}$, at $60{ }^{\circ} \mathrm{C}$ after $30 \mathrm{~min}$, and at $70{ }^{\circ} \mathrm{C}$ after $10-15$ min of reaction performing.

The graph showing the yield of ECDD versus reaction time (Fig. 6) is more difficult to interpret because one dominant trend can not be seen. At the temperature of $40{ }^{\circ} \mathrm{C}$, the yield of ECDD increases to $46 \mathrm{~mol} \%$ for the reaction time to $30 \mathrm{~min}$, then it decreases to $42.4 \mathrm{~mol} \%$ (after the reaction time of $60 \mathrm{~min}$ ). At the temperature of $50{ }^{\circ} \mathrm{C}$ the value of the yield of ECDD also increases to $30 \mathrm{~min}$, but at this temperature it reaches the highest value of all- $53.2 \mathrm{~mol} \%$ and then decrease to $45 \mathrm{~mol} \%$ while continuing the process. At $60{ }^{\circ} \mathrm{C}$, the yield of ECDD increases to $34.1 \mathrm{~mol} \%$ for the reaction time to $15 \mathrm{~min}$, after which it decreases to about $30 \mathrm{~mol} \%$ and remains constant until the end of the reaction. At the temperature $70{ }^{\circ} \mathrm{C}$, the maximum of the yield of ECDD is probably reached for the reaction time shorter than $5 \mathrm{~min}$. At this temperature, the yield of ECDD steadily decreases in the range of 5-15 min reaching the value of about $38 \mathrm{~mol} \%$ and remains at this level until the end of the reaction. Among the tested temperatures, the best turned out to be $50{ }^{\circ} \mathrm{C}$, at which the maximum of the yield of ECDD was $53.2 \mathrm{~mol} \%$ after $30 \mathrm{~min}$ of reaction carrying out.

It is worth noting that in a two-phase PTC system, temperature affects the kinetics of the reaction in many different ways. It is possible to increase the reaction rate in the organic phase by increasing the energy of the reagents, as in single-phase 
reactions [27]. However, one should not forget that the mutual solubility of the components of the reaction mixture depends on the temperature and the composition of the reaction mixture is complex and changes during the course of the reaction. Initially, the organic phase contains only CDT and solvent, and as the reaction progresses, ECDD as well as diECDD also appears in it. This obviously increases the polarity of the organic phase. It should also be remembered that the PTC mechanism is complex and there are still doubts as to the actual course of this reaction. The first hypothesis assumes the existence of an interfacial area into which the reagents are transported and in which the reaction takes place. The second is the extraction mechanism, which assumes that reagents in the form of lipophilic ionic pairs are transported from the inside of one phase to the other [28].

Indeed, independent of the real reaction mechanism, the increase in temperature should affect well on the accelerating of the reaction course. In case of the first mechanism, the increase in temperature is the key to accelerating the reaction by reducing the size of the micelles, resulting in increasing their number and, consequently, increasing in the total reaction surface. In case of the second mechanism, the increase in temperature may cause changes in the partition coefficient of the catalyst (its oxidized and reduced form) between the aqueous and organic phases, and smaller micelles additionally facilitate mass exchange between the phases.

The conversion of hydrogen peroxide was independent of the temperature of the process, the only exception was the reaction carried out at $40{ }^{\circ} \mathrm{C}$, at which its value was much lower than the others. Conversions of hydrogen peroxide at 50, 60 and $70{ }^{\circ} \mathrm{C}$ were about $99 \mathrm{~mol} \%$. At the temperature of $40{ }^{\circ} \mathrm{C}$, the conversion of hydrogen peroxide was 12 percentage points lower than at $50{ }^{\circ} \mathrm{C}$, with the conversion of CDT differing by 3.7 percentage points, which may indicate that hydrogen peroxide decomposition reactions occur at higher temperatures.

\section{Influence of solvent type}

In order to study the effect of solvent type on the results of the CDT epoxidation, reactions were carried out using three different solvents: toluene, chloroform and ethyl acetate (EtOAc), as well as for the following process parameters: temperature $50{ }^{\circ} \mathrm{C}$, solvent content $50 \mathrm{wt} \%$, catalyst content $0.55 \mathrm{~mol} \% \mathrm{CDT}$ and the molar ratio $\mathrm{H}_{2} \mathrm{O}_{2}$ :CDT 1.5:1. Chloroform and toluene were used because of their frequency of use in oxidation reactions under PTC conditions. Chloroform is used even in the Ishii catalytic system [29], and toluene in the Sato catalytic system [30]. Ethyl acetate was used because of its lower environmental impact in comparison to toluene and chloroform, and there are no reports about its use in oxidation reactions under PTC conditions. Table 4 and Fig. 7 show the results of studies involving selected solvents.

The conversions of CDT for ethyl acetate and chloroform were very similar throughout the duration of the reaction, slight differences only occurred at $30 \mathrm{~min}$ and disappeared at $60 \mathrm{~min}$. For both of these solvents, the conversions of CDT were higher than those obtained with toluene throughout the entire duration of the reaction. 
Table 4 The values of the conversion of the conversion of CDT (the values of the yields of ECDD are also given in brackets) for reactions carried out with different solvents ${ }^{a}$

\begin{tabular}{|c|c|c|c|}
\hline \multirow[t]{2}{*}{ Time (min) } & \multicolumn{3}{|l|}{ Solvent } \\
\hline & Toluene & $\mathrm{CHCl}_{3}$ & EtOAc \\
\hline 0 & $0(0)$ & $0(0)$ & $0(0)$ \\
\hline 5 & $35.6(26.1)$ & $45.8(31.1)$ & $46.2(33.8)$ \\
\hline 10 & $48.6(37.2)$ & $62.1(39.5)$ & $62.9(42.3)$ \\
\hline 15 & $59.5(47.0)$ & $75.0(44.2)$ & $73.4(44.1)$ \\
\hline 30 & $83.3(53.2)$ & $94.8(36.9)$ & 88.0 (41.9) \\
\hline 60 & $93.3(45.0)$ & $95.5(34.4)$ & 93.9 (34.7) \\
\hline $60^{[\mathrm{b}]}$ & 98.9 & 99.4 & 99.6 \\
\hline
\end{tabular}

${ }^{\text {a }}$ The reaction conditions were the same as those given in the title in Fig. 4

${ }^{\mathrm{b}}$ The values of conversion of $\mathrm{H}_{2} \mathrm{O}_{2}$
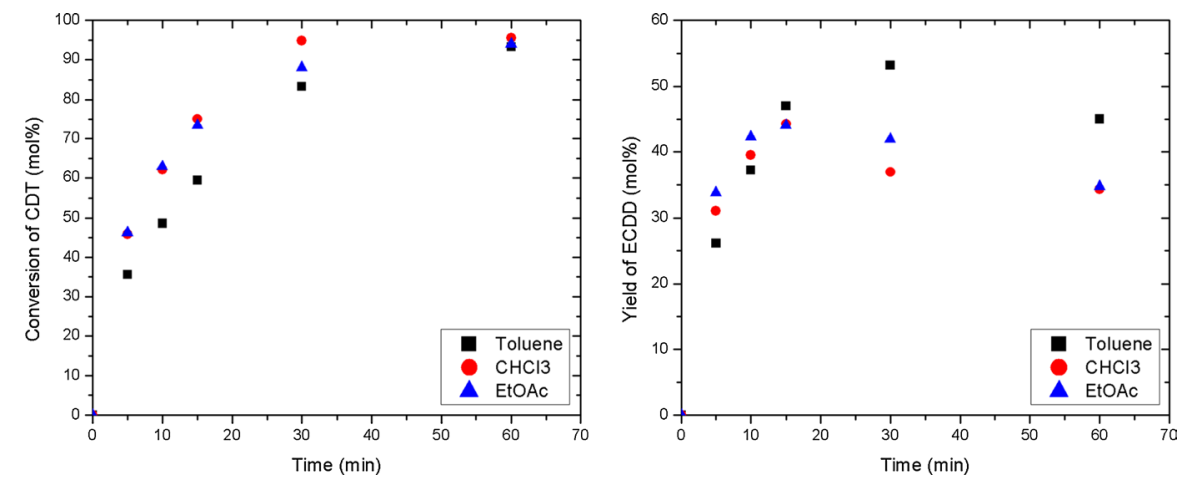

Fig. 7 Drawing showing the conversion of CDT and the yield of ECDD as a function of time for reactions with different solvents. Reaction conditions were the same as those given in the title in Fig. 4

The largest differences in the yield of ECDD between toluene and other solvents can be seen after 30 and $60 \mathrm{~min}$. After this time, the yield of ECDD using it is more than 10 percentage points higher than using the other two solvents.

The higher yield of ECDD for the reaction carried out using toluene may result from its hydrophobicity, expressed by the polarity, whose value is lower than the values for chloroform and ethyl acetate (0.099; 0.259; 0.228 [31]). Lower polarity limits water availability and, consequently, inhibits the epoxy ring hydrolysis reaction. After the reaction was completed, almost complete conversion of hydrogen peroxide was obtained, regardless of the solvent used.

In this series of studies, the tendency to formation of emulsion was the feature that differentiated individual syntheses. In the case of toluene, the emulsion practically did not form, for ethyl acetate the formation of emulsion was noticeable, however, it disappeared after about 10-15 s after stopping of mixing, for chloroform the emulsion 
formation intensified with the passage of time of reaction and after $30 \mathrm{~min}$ of reaction the emulsion did not separate even after waiting of $30 \mathrm{~s}$ after stopping of mixing.

The similarity of changes in the conversion of CDT for reactions carried out using $\mathrm{CHCl}_{3}$ and EtOAc can be attempted to explain the similar polarity of these solvents (0.259 and 0.228 , respectively), which is much higher than the polarity of toluene (0.099). The increased polarity of these solvents improves their solubility in water. Their increased content in the aqueous phase may in turn improve the solubility of the catalyst and facilitate the oxidation of its reduced form.

\section{Conclusion}

The possibility of epoxidation of CDT with $\mathrm{H}_{2} \mathrm{O}_{2}$ was investigated in the new catalytic system consisting of $\mathrm{H}_{2} \mathrm{WO}_{4}, \mathrm{H}_{3} \mathrm{PO}_{4}$ and $\left[\mathrm{CH}_{3}\left(\mathrm{CH}_{2}\right)_{7}\right]_{3} \mathrm{CH}_{3} \mathrm{~N}^{+} \mathrm{HSO}_{4}{ }^{-}$, mixed together in the molar ratio 2:1:1 and under different reaction conditions. The influence of catalyst content, the molar ratio $\mathrm{H}_{2} \mathrm{O}_{2}$ : CDT, temperature and type of solvent on the course of studied reaction was examined. The described catalyst system proved to be active in the epoxidation of CDT with hydrogen peroxide. The change of each of the examined process parameters affected the results of the conducted reaction, while each of the parameters affected the results of the reaction to a different degree. The highest yield of ECDD throughout the entire study was obtained for the reaction carried out using the catalyst content $0.45 \mathrm{~mol} \% \mathrm{CDT}$, the molar ratio $\mathrm{H}_{2} \mathrm{O}_{2}$ : CDT 1.5:1 and toluene as the solvent after $30 \mathrm{~min}$ of reaction carrying out. It was equal to $54.9 \mathrm{~mol} \%$.

For most reactions, there is a noticeable decrease in the value of the yield of ECDD after exceeding by the conversion of CDT value of $70 \mathrm{~mol} \%$. This is most likely associated with further oxidation of ECDD to diECDD.

In a series of studies to investigate the effect of the molar ratio of $\mathrm{H}_{2} \mathrm{O}_{2}$ :CDT on the results of CDT epoxidation, it was found that the amount of hydrogen peroxide does not affect the initial reaction rate, however, the excess of oxidant adversely affects the yield of ECDD in the later stages of the reaction.

The similarity of the time course of CDT epoxidation reactions for the tested solvents indicates that the type of solvent only slightly affects the results of the reaction. Therefore, it is possible to replace chloroform with more environmentally friendly and safer ethyl acetate, without significantly reducing the efficiency of the process.

In the course of further research on the process of epoxidation of CDT with hydrogen peroxide using the catalytic system consisting of $\mathrm{H}_{2} \mathrm{WO}_{4}, \mathrm{H}_{3} \mathrm{PO}_{4}$ and methyltrioctylammonium hydrogen sulfate, further research should be carried out to determine the impact of other relevant process parameters, followed by their optimization. Considering the numerous applications of ECDD, further research on this process is desirable.

Open Access This article is licensed under a Creative Commons Attribution 4.0 International License, which permits use, sharing, adaptation, distribution and reproduction in any medium or format, as long as you give appropriate credit to the original author(s) and the source, provide a link to the Creative Commons licence, and indicate if changes were made. The images or other third party material in this article are included in the article's Creative Commons licence, unless indicated otherwise in a credit line to the material. If material is not included in the article's Creative Commons licence and your intended use is 
not permitted by statutory regulation or exceeds the permitted use, you will need to obtain permission directly from the copyright holder. To view a copy of this licence, visit http://creativecommons.org/licen ses/by/4.0/.

\section{References}

1. Akiike J (2016) Slurry for lithium ion secondary battery porous film, production method therefor, separator for lithium ion secondary battery, and lithium ion secondary battery. US Patent App 2016/0,013,465, 2016

2. Fukumine M, Yamamoto N (2018) Composition for electrochemical device electrode, electrode for electrochemical device, and method of producing composition for electrochemical device electrode. US Patent App 2018/0,301,744, 2018.

3. Toyoda Y, Sasaki T (2018) Separator for secondary cell. US Patent 9,941,497, 2018.

4. Tabuchi M, Miura K, Miyashiro H, Bobayashi Y (2016) Positive electrode and nonaqueous electrolyte secondary battery. US Patent App 2016/0,006,075, 2016

5. Matsubayashi S, Suzuki K (2018) Conductive polymer solution, capacitor, and method for producing the capacitor. US Patent App 2018/0,334,577, 2018.

6. Fujiki H, Matsubayashi S, Kanto K, Suzuki F (2015) Curable antistatic organopolysiloxane composition and antistatic silicone film. US Patent App 2015/0,348,670, 2015.

7. Fujiki H, Suzuki T (2015) Conductive composition, conductive composition production method, anti-static resin composition and antistatic resin film. US Patent App 2015/0,348,671, 2015.

8. Emori N, Ogawa T, Arakawa T (2018) Cross-linked rubber product. US Patent App 2018/0,030,216, 2018.

9. Komoriya H, Suzuki K, Tanaka T, et al (2015) Antibacterial agent, substrate surface treatment method using the same, antibacterial agent composition, and substrate surface treatment method using the same. US Patent 9,204,652, 2015.

10. Fankhauser P (2017) Unsaturated macrocyclic epoxide as perfuming ingredient. US Patent App 2017/0,058,236, 2017.

11. Fankhauser $P$ (2016) Cyclododecadienone derivatives as perfuming ingredients. US Patent App 2016/0,060,569, 2016.

12. Lewandowski G, Gruszkowska E, Milchert E (2005) The influence of the solvent on the epoxidation of cis, trans, trans-1,5,9-cyclododecatriene to trans-1,2-epoxy-cis, trans-5,9-cyclododecadiene by catalytic system tert-butyl hydroperoxide/Mo(CO) 6 . J Hazard Mater 120:45-50. https:// doi.org/10.1016/j.jhazmat.2004.12.032

13. McKeen LW (2010) Polyamides (nylons). In: McKeen LW (ed) Fatigue and tribological properties of plastics and elastomers, 2nd edn. Elsevier, Oxford, pp 175-228

14. (2012) Evonik says at least 3 months to normal CDT output. https://www.reuters.com/article/ evonik/evonik-says-at-least-3-months-to-normal-cdt-output-idUSL2E8FHBNB20120417. Accessed 9 Dec 2019

15. Evans S (2012) Fire in small German town could curb world car production. https://www.bbc. com/news/business-17769466. Accessed 9 Dec 2019

16. Sheffi Y (2018) Modeling risks in supply chains. In: Gong S, Cullinane K (eds) Finance and risk management for international logistics and the supply chain. Elsevier, San Diego, pp 55-84

17. Li JJ, Corey EJ (2010) Oxidation. In: Li JJ, Corey EJ (eds) Name reactions for functional group transformations. Wiley, Hoboken, pp 159-332

18. Wolfgang E, Gunter, R. S (1989) Process for production of epoxides. US Patent 4,882,442, 1989.

19. Nobuyuki K, Mitsuo Y, Hirofumi T, Junichi K (2001) Process for producing epoxycyclododecadiene. US Patent 6,172,243 B1, 2001

20. Hubert M (2009) Process for the preparation of lactones or epoxides. US Patent 7,528,268, 2009

21. Lewandowski G, Rytwińska E, Milchert E (2006) Sposób otrzymywania 1,2-epoksy-(5Z,9E)5,9-cyklododekadienu. PL Patent 213,052

22. Lewandowski G, Milchert E (2007) Sposób epoksydacji (1Z,5E,9E)-1,5,9-cyklododekatrienu do 1,2-epoksy-(5Z,9E)-5,9-cyklododekadienu. PL Patent 212,327.

23. Mahajan SS, Sharma MM, Sridhar T (2007) Uncatalyzed oxidation of 1,5,9-cyclododecatriene with molecular oxygen. Ind Eng Chem Res 46:3057-3062. https://doi.org/10.1021/ie061072n 
24. Nobuyuki K, Mitsuo Y, Osamu Y et al (2000) Process for producing 1,2-epoxy-5,9-cyclododecatriene. US Patent 6,043,383, 2000.

25. Lewandowski G, Milchert E, Sałaciński Ł (2016) Sposób otrzymywania 1,2-epoksy-5,9-cyklododekadienu; PL Patent 233233 B1

26. Lewandowski G (2013) Efficiency of selected phase transfer catalysts for the synthesis of 1,2-epoxy-5,9-cyclododecadiene in the presence of $\mathrm{H}_{2} \mathrm{O}_{2} / \mathrm{H}_{3} \mathrm{PW}_{12} \mathrm{O}_{40}$ as catalytic system. Pol J Chem Technol 15:96-99. https://doi.org/10.2478/pjct-2013-0053

27. Schwaab M, Pinto JC (2007) Optimum reference temperature for reparameterization of the Arrhenius equation. Part 1: Problems involving one kinetic constant. Chem Eng Sci 62:2750 2764. https://doi.org/10.1016/j.ces.2007.02.020

28. Panicheva LP, Meteleva GP, Ageikina OV, Panichev SA (2018) Phase transfer of the organic substrate in the epoxidation reaction of allyl chloride in two-phase aqueous-organic systems. Pet Chem 58:884-888. https://doi.org/10.1134/S0965544118100146

29. Ishii Y, Yamawaki K, Ura T et al (1988) Hydrogen peroxide oxidation catalyzed by heteropoly acids combined with cetylpyridinium chloride. Epoxidation of olefins and allylic alcohols, ketonization of alcohols and diols, and oxidative cleavage of 1,2-diols and olefins. J Org Chem 53:3587-3593. https ://doi.org/10.1021/jo00250a032

30. Sato K, Aoki M, Ogawa M et al (1996) A practical method for epoxidation of terminal olefins with 30\% hydrogen peroxide under halide-free conditions. J Org Chem 61:8310-8311. https://doi. org/10.1021/jo961287e

31. Reichardt C, Welton T (2010) Empirical parameters of solvent polarity. In: Reichardt C, Welton T (eds) Solvents and solvent effects in organic chemistry, 4th edn. Wiley-VCH Verlag GmbH \& Co. KGaA, Weinheim, pp 425-508

Publisher's Note Springer Nature remains neutral with regard to jurisdictional claims in published maps and institutional affiliations. 